\title{
Dificultades para la detección de personas con discapacidad intelectual y psicosocial detenidas por la policía en la Ciudad de Buenos Aires
}

\author{
Difficulties in detecting people with intellectual and psychosocial \\ disabilities detained by the police in the City of Buenos Aires
}

\begin{abstract}
Resumen
Las personas con discapacidad intelectual y psicosocial se encuentran sobrerrepresentadas en el ámbito de la justicia penal. La falta de identificación adecuada en las primeras etapas suele perdurar a lo largo de todo el proceso penal. Objetivo: determinar qué cantidad de personas con discapacidad intelectual o psicosocial que se encontraban detenidas sospechosas de la comisión de un delito fueron detectadas por parte del personal médico de la policía en la Ciudad de Buenos Aires. Materiales y métodos: estudio observacional de tipo retrospectivo de 70 casos de personas detenidas. Resultados: la muestra analizada fue de 47 casos de personas detenidas. De estas, 29 presentaban diagnóstico de discapacidad intelectual y 18 de discapacidad psicosocial. El personal médico de la policía realizó 61 evaluaciones. Sólo en el $8 \%$ de las evaluaciones se detectó discapacidad. En ninguno caso se implementaron apoyos y/o ajustes en el procedimiento. Se deben implementar estrategias para mejorar la detección temprana de las personas con discapacidad sometidas a un proceso penal.
\end{abstract}

\section{Palabras clave}

Discapacidad intelectual; discapacidad psicosocial; detección; detención policial.

\begin{abstract}
People with intellectual and psychosocial disabilities are overrepresented in criminal justice. The lack of an adequate identification in the early stages usually lasts throughout criminal processes. Objective: to determine how many people with intellectual or psychosocial disabilities who were detained suspected of committing a crime were detected by the police medical staff in the City of Buenos Aires. Materials and methods: retrospective observational study of 70 cases of detainees. Results: the sample studied was of 47 detainees. Of those, 29 had a diagnosis of intellectual disability and 18 of psychosocial disability. Police medical staff conducted 61 evaluations. Only in $8 \%$ of the evaluations carried out, a disability was detected. In no case supports and / or adjustments were implemented in the criminal procedure. Strategies to improve early identification of people with disabilities in criminal justice should be implemented.
\end{abstract}

\section{Keywords}

Intellectual disability; psychosocial disability; identification; police custody.

\section{Ezequiel N. Mercurio \\ <ezequielmercurio@gmail.com>}

Centro Interdisciplinario de Investigaciones Forenses de la Academia Nacional de Ciencias de Buenos Aires. Argentina

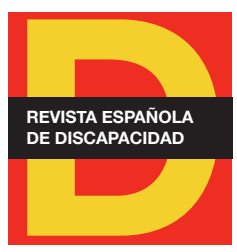

Para citar:

Mercurio, E. N. (2021). Dificultades para la detección de personas con discapacidad intelectual y psicosocial detenidas por la policía en la Ciudad de Buenos Aires. Revista Española de Discapacidad, 9(1), pp. 121-140.

Doi: <https://doi.org/10.5569/23405104.09.01.06>

Fecha de recepción: 25-11-2019 Fecha de aceptación: 12-03-2021 


\section{Introducción ${ }^{1}$}

Diferentes publicaciones internacionales señalan que las personas con discapacidad intelectual y psicosocial (World Network of Users and Survivors of Psychiatry, 2008, p.12) ${ }^{2}$ se encuentran sobrerrepresentadas en el ámbito de la justicia penal. Dependiendo de la metodología utilizada para el diagnóstico de discapacidad intelectual se ha reportado una prevalencia entre $0.5 \%$ y el $10.8 \%$ (Fazel et al., 2008; Søndenaa et al., 2008) mientras que en la población general la prevalencia es del $1 \%$. Cifras entre $4 \%$ y $6.7 \%$ han sido reportadas por otros estudios internacionales (Hassiotis et al., 2011) y hasta el $30 \%$ si se tomaban definiciones más amplias como trastorno en el aprendizaje (Loucks, 2007). Un reciente estudio realizado en cárceles españolas reportó que el $3.77 \%$ de las personas evaluadas tenía un $\mathrm{CI}(\mathrm{IQ})<70$ y dicho porcentaje se elevaba al $11 \%$ se si tomaban valores límites de Cl (Tort et al., 2016). Por su parte otro estudio realizado publicado en 2017 sobre la prevalencia de las personas con discapacidad intelectual en las cárceles del Reino Unido reportó que el $11 \%$ de los reclusos presentaba una discapacidad intelectual (Chaplin et al., 2017) En tanto que en Irlanda se estimó que el $28 \%$ de la población penitenciaria presenta una discapacidad intelectual (Gulati et al., 2018) En un estudio realizado ya hace 25 años Lyall y colaboradores hallaron que el 11,5\% de las personas detenidas en un lapso de tres meses en una Comisaría de Londres había concurrido a una escuela especial (Lyall et al., 1995).

Con relación a la discapacidad psicosocial investigaciones recientes han reportado una prevalencia cercana al $50 \%$ de las personas privadas de libertad en la cárcel (Al-Rousan et al., 2017; James y Glaze, 2006; United Nations Office on Drugs and Crime, 2009). Asimismo, un estudio realizado en tres cárceles españolas halló que la prevalencia de trastorno mental a lo largo de la vida fue del $90.2 \%$, siendo el trastorno más común el consumo problemático de sustancias (72.3\%) (Zabala-Baños et al., 2016).

Un estudio en realizado España por el Observatorio Estatal de la discapacidad publicado en el 2018 reportó que dentro de las personas con discapacidad que se encuentran en prisión la discapacidad intelectual y psicosocial son las más frecuente $(53,83 \%$ en el primer caso) (Observatorio estatal de la discapacidad, 2018, p. 49)

En el ámbito español el interés por conocer la situación de las personas con discapacidad intelectual en prisión se remonta a la década del ochenta (Ramos Feijóo, 2011, p. 8); Argentina por su parte aún no cuenta con estadísticas precisas y actualizadas sobre la situación de las personas con discapacidad intelectual y psicosocial que se hayan sometidas a un proceso penal. Con el objetivo tener un primer acercamiento sobre la situación de las personas con discapacidad en las cárceles argentinas, el Programa Nacional de Asistencia para las Personas con Discapacidad en sus Relaciones con la Administración de Justicia (ADAJUS), llevó adelante en el 2012 una encuesta en 3 cárceles. Se censaron 3578 personas, de las cuales 328 reportaron presentar alguna discapacidad. El $10 \%$ correspondía a personas con discapacidad intelectual y el $22 \%$ a personas con discapacidad mental (Equipo Adajus, 2012; 2013). Se trató de un censo que utilizó

1. Agradecimientos: agradezco a mis colegas Laura Bermolen, Damian Aloia, Alba Ayala y Aldana Hosni que han contribuido en la recolección de los datos.

2. A lo largo del presente se utilizará el término persona con discapacidad psicosocial para referirse a aquellas personas que presentan una discapacidad por presentar una deficiencia mental a las que se refiere el artículo 1 de la Convención sobre los Derechos de las Personas con Discapacidad. Usualmente en los ámbitos de salud se utiliza el término personas con enfermedades o padecimientos mentales. Se ha preferido utilizar el término discapacidad psicosocial ya que permite expresar la interacción entre las barreras sociales y culturales y el sujeto que presenta un padecimiento psíquico. 
una batería de preguntas propuestas por el Grupo de Washington, motivo por el cual los datos arrojados deben ser interpretados con prudencia, ya que se trata de una metodología de detección que depende de la percepción que tiene cada entrevistado de sí mismo ${ }^{3}$. Por otra parte, dentro del penitenciario federal se lleva adelante desde el año 2011 el Programa Interministerial de Salud Mental Argentino (PRISMA) con prestaciones similares a las de un hospital psiquiátrico penitenciario, ya que allí se realizan las internaciones por motivos de salud mental de aquellos internos que requieren dicha modalidad de abordaje y donde se encuentran las personas con medida de seguridad. Dicho programa presenta criterios estrictos de admisión basados en el principalmente en el diagnóstico. Dichos diagnósticos involucran personas con cuadros de psicosis, excitación psicomotriz y retraso mental de moderado a profundo, pero no se admiten personas con retraso mental leve, trastornos de la personalidad, trastornos por consumos de sustancias (Ministerio de Justicia y Ministerio de Salud, 2011). EI PRISMA cuenta con 60 plazas para hombres, 10 para mujeres y 10 para observación. Lamentablemente no se han hallado datos publicados desagregados sobre la cantidad de personas que allí se encuentran admitidas, padecimientos que presentan, condición legal, tiempo de internación, etc.

La Convención sobre los Derechos de las Personas con Discapacidad (en adelante la Convención) señala en su artículo 13 que los Estados deben garantizar el acceso a la justicia en igual de condiciones en todas las etapas e instancias de los procesos judiciales, esto incluye las primeras fases de la detención por parte de las fuerzas policiales (Naciones Unidas, 2008). Para ello deben adoptarse ajustes de procedimiento y garantizarse los apoyos que se requieran para el ejercicio de la capacidad jurídica (Naciones Unidas, 2020). Asimismo, la Convención señala que, con el objetivo de cumplir con un real acceso a la justicia, deben realizarse actividades de capacitación de las personas que trabajan en la administración de justicia, incluyendo personal penitenciario y de las fuerzas de seguridad (Naciones Unidas, 2008).

Los agentes de las fuerzas de seguridad y el personal médico de la policía son los primeros en intervenir en una detención. Es por ello que recientemente Naciones Unidas ha reafirmado y resaltado la importancia de la capacitación, formación y sensibilización en el modelo de derechos humanos de la discapacidad y establecer buenas prácticas en el acceso a la justicia de los primeros intervinientes (Naciones Unidas, 2020). Así, la implementación de ajustes de procedimientos y de apoyos para el ejercicio de la capacidad jurídica requieren en primer lugar de una adecuada identificación de las personas con discapacidad intelectual y psicosocial que se encuentran sometidas a un proceso penal (Recover y Araoz, 2014; Sheinbaum y Vera, 2016, p. 162; Plena Inclusión, 2018). Sin embargo, diversas publicaciones han señalado que las personas con discapacidad intelectual en el ámbito de la justicia penal se encuentran fuertemente invisibilizadas (Mercurio, 2016a; Mercurio, 2016b; Irish Penal Reform Trust, 2020; Plena Inclusión, 2020)

Las personas con discapacidad intelectual que se encuentran sometidas a un proceso penal, son altamente vulnerables y en general han atravesado múltiples privaciones a lo largo de su vida. En este sentido tal como lo señala Ramos Feijóo (2013) cabe preguntarse qué ha sucedido con las redes de apoyos; la presencia de personas con discapacidad en el ámbito penitenciario pone de manifiesto el fracaso o la directamente ausencia de las estrategias de integración (Ramos Feijóo, 2013, p. 17).

3. El Grupo de Washington se creó en el 2001 en el marco de las Naciones Unidas con el objetivo de promover y coordinar la cooperación internacional en la medición de la discapacidad y adecuar herramientas para los censos y las encuestas locales. En este marco el Grupo ha desarrollado encuestas y cuestionarios basados en la Clasificación Internacional de Funcionamiento (CIF). Para más información ver https://www. washingtongroup-disability.com acceso el 20 de noviembre de 2020 
Se trata de un grupo particular de alta vulnerabilidad, en franca desventaja, excluido e invisibilizado. Se ha reportado que las personas con discapacidad acusadas de un delito tienen menos años de educación, se encuentran desempleadas, han padecido diferentes formas de maltrato y abandono durante su infancia, tienen dificultades en sus habilidades sociales, problemas para comunicarse y comprender, y trastornos en su comportamiento (Crocker et al., 2007). Los hogares de personas con discapacidad son más pobres que aquellos que no tienen discapacidad. "Las personas con discapacidad obtienen resultados más deficientes en el ámbito educativo y en el mercado laboral y tienen más probabilidades de ser pobres que las personas sin discapacidad (Organización Mundial de la Salud, 2011, p. 44). Tal como lo señala Lorenzo García (2015) el fracaso de los sistemas de apoyo, social, de salud, y educativo para las personas con discapacidad intelectual, también incluye un fracaso a lo largo de la vida de la familia. Es por ello que la detección temprana de casos de discapacidad intelectual y la identificación de situaciones de riesgo a las que se encuentra expuesta la persona y su familia son medidas de prevención que deben fortalecerse (Sánchez Miranda y Bayona Juan, 2019).

Desde hace más de dos décadas, este grupo particularmente vulnerable ha recibido gran atención en el ámbito de la justicia penal en diferentes países, y sobre todo en aquellos países donde la policía tiene la facultad de interrogar a las personas detenidas, dado que se ha detectado que las personas con discapacidad sometidas a la justicia penal tienen dificultades para comprender sus derechos, son más sugestionables, más aquiescentes, y pueden auto incriminarse a través de confesiones falsas (Howard y Tyrer, 1998).

En este contexto, se han desarrollado diferentes medidas de salvaguarda. Por ejemplo, en algunos sistemas penales se prevé la participación en el interrogatorio de una tercera persona independiente (Villamanta Disability Rights Legal Service, 2012). Estas medidas requieren en primer lugar de la detección temprana. En este mismo sentido, desde hace varios años el Programa Reclusos de Plena Inclusión (antes FEAPS) viene realizando con “...diferentes operadores jurídicos (...) cursos de formación, sensibilización y capacitación, con el fin de facilitar herramientas para la detección y el apoyo de personas con discapacidad intelectual o del desarrollo bien en calidad de víctimas, testigos o autores” (Recover y Araoz, 2014, p. 28).

La policía es el primer punto de contacto entre una persona con discapacidad acusada de una infracción penal y la justicia penal, por tal motivo sus intervenciones resultan de vital importancia y marcaran en la mayoría de los casos el camino de esas personas (Bradley, 2009). En este sentido, las intervenciones que realice la policía en estas primeras etapas son cruciales, ya que brindarán información a los diferentes actores judiciales en las etapas posteriores del proceso (Bradley, 2009) En esta misma línea, Recover y Araoz (2014) señalan "la necesidad, incluso la obligatoriedad de que las Fuerzas de Seguridad cuenten con la formación precisa para detectar y aplicar los ajustes necesarios ante la sospecha o indicación de una discapacidad... (d)e esta forma, en caso de sospecha inicial, bien en la misma Comisaria o cuerpo policial correspondiente, Puesto de la Guardia Civil o Juzgado, se podría obtener este dato esencial para orientar tanto la declaración como el resto de actuaciones penales subsiguientes" (p. 46).

Por lo expuesto, la falta de una identificación adecuada de una persona con discapacidad intelectual o psicosocial que se encuentra detenida, se erige como la primera barrera para el acceso a la justicia ${ }^{4}$ (Loucks, 2007).

4. El informe Bradley, al igual muchas publicaciones provenientes del Reino Unido, se refieren a dificultades en el aprendizaje, no se refiere a discapacidad intelectual y los define como una disminución significativa para comprender información nueva o compleja, para aprender nuevas habilidades (déficit en la inteligencia) asociado a una dificultad para hacer frente a una vida independiente (déficit en el funcionamiento social), que comienza antes de la vida adulta, con efectos duraderos en el desarrollo. La categoría trastorno del aprendizaje incluye a una amplia gama de entidades clínicas que van desde la dislexia hasta afecciones del neurodesarollo como Síndrome de Asperger. En el presente trabajo las referencias que artículos citados realicen sobre trastornos en el aprendizaje serán homologadas como discapacidad intelectual, teniendo en cuenta que dentro de la definición amplia de trastorno del aprendizaje que dichos trabajos realizan, se encuentra la discapacidad intelectual. 
La falta de identificación adecuada en las primeras etapas suele perdurar en muchos casos a lo largo del todo el proceso ${ }^{5}$. Pearse (1991), citado por Pearse (1995), señaló hace más de tres décadas que el fracaso de los policías en identificar las potenciales vulnerabilidades psicológicas de los sospechosos representaba una amenaza real para las bases fundantes del sistema legal inglés. En este sentido, el Informe Bradley destacó, ya hace una década, la falta de entrenamiento adecuado de los policías en temáticas de salud mental y discapacidad intelectual (Bradley, 2009). En Australia reportaron problemáticas similares con relación las dificultades de la policía para identificar a las personas con discapacidad intelectual (Hayes, 2000 citado por Villamanta, 2012).

Si bien el Informe Bradley realizó recomendaciones específicas y puntuales con el objetivo de mejorar la detección, identificación y tratamiento de las personas con discapacidad intelectual o psicosocial en el ámbito penal, investigaciones posteriores mostraron resultados negativos con relación a la situación de personas con discapacidad intelectual que son arrestadas por la policía (Criminal Justice Joint Inspection, 2014). En este sentido el informe realizado en el 2014 por la Criminal Justice Joint Inspection señaló su decepción luego del informe Bradley del 2009 al encontrar solo unos pocos los y las profesionales de la medicina o psiquiatras entrenados para trabajar con personas con discapacidad intelectual en las comisarías, y resaltaron que se trató de una oportunidad perdida para derivar a muchas personas con discapacidad a servicios más apropiados y acordes o al menos para asegurarse que las personas con discapacidad intelectual sean tratadas de manera adecuada por la justicia penal (Criminal Justice Joint Inspection, 2014, p. 2).

El informe realizado por la Criminal Justice Joint Inspection (2014) mostró que la detección de personas con discapacidad intelectual en las primeras etapas del proceso era muy pobre. El $58 \%$ de las personas con discapacidad intelectual no habían sido detectadas, a pesar de que algunas de ellas presentaban indicadores robustos para sospechar una discapacidad intelectual, como por ejemplo problemas para leer y escribir, o inclusive algunos de ellos habían asistido a una escuela especial (p.18).

En el medio latinoamericano un reciente trabajo, que comparó una muestra de 40 personas con discapacidad intelectual y psicosocial provenientes de la Ciudad de Buenos Aires (20 casos) y la Ciudad de México (20 casos) que fueron imputadas por la probable comisión de un delito, mostró una detección temprana muy pobre por parte del personal médico de policía. En el caso de México sólo en 4 de los 20 casos la discapacidad fue identificada y en el caso de la Ciudad de Buenos Aires, en ninguno de los 20 casos se identificó. En el caso de la Ciudad de Buenos Aires, 8 de las 20 personas detenidas tenía certificado de discapacidad y en el caso de la Ciudad de México, 13 tenían evaluaciones previas que daba cuenta de su discapacidad (Bernardini et al., 2018).

Con el objetivo de asegurar un trato adecuado a las personas con discapacidad que se encuentran detenidas, se han desarrollado guías específicas para personal policial para mejorar la detección temprana (The National Autistic Society, 2008; 2015; National Policing Improvement Agency \& Association of Chief Police Officers, 2010). En el ámbito español, el Programa de personas con discapacidad intelectual y del desarrollo en el ámbito de la justicia penal llevado adelante por Plena Inclusión desde la década del ochenta ha desarrollado múltiples publicaciones relacionadas con el acceso a la justicia (Araoz Sánchez-Dopico, 2020), documentos sobre la prisión en lectura fácil (Rodríguez, 2019). En este sentido, resulta de vital importancia

5. La Asociación Americana sobre Discapacidad Intelectual y del Desarrollo, señala que las personas con discapacidad intelectual y del desarrollo son frecuentemente no diagnosticadas o mal diagnosticadas y que se les niegan evaluaciones adecuadas en base a estereotipos falsos sobre lo que pueden o no pueden hacer. American Association on Intellectual and Developmental Disabilities. Criminal Justice System disponible en https:// www.aaidd.org/news-policy/policy/position-statements/criminal-justice 
crear salvaguardas, generar mecanismos de apoyos para personas con discapacidad intelectual y psicosocial que se encuentran detenidas, teniendo en cuenta que se trata de un colectivo vulnerable y que muchas veces, como señala Talbot, intentan esconder su condición por vergüenza y miedo de hacer el ridículo (Talbot, 2011; Loucks y Talbot, 2007).

Las personas con discapacidad pueden no comprender de forma adecuada las instancias del proceso penal, pueden auto incriminarse, realizar confesiones falsas, además de encontrarse expuestos a situaciones violencia, maltrato y abusos dentro de la prisión. En este sentido se destacan la experiencia en el ámbito mexicano sobre facilitadores en el sistema de justicia llevada adelante por la organización civil Documenta (Lizama y Sheinbaum, 2020) y en Argentina el Programa Nacional de Asistencia para las Personas con Discapacidad en sus Relaciones con la Administración de Justicia, ambos en consonancia con las más recientes recomendaciones realizadas por la ex Relatora Especial de Discapacidad de Naciones Unidas (Naciones Unidas, 2020). En esa misma línea también lo señala Plena Inclusión en su informe sobre la persona facilitadora en procesos judiciales (Plena Inclusión, 2020)

En la Ciudad de Buenos Aires toda persona que es detenida por las fuerzas de seguridad sospechosa de haber cometido un delito debe ser evaluada por profesionales de la salud pertenecientes a dicha fuerza de seguridad con el objetivo de verificar su estado físico y mental al momento de su aprehensión ${ }^{6}$. Dicha evaluación se realiza en las Comisarías o Alcaidías durante las horas posteriores a la detención. El personal de salud encargado de dichas evaluaciones son dependientes de las fuerzas de seguridad, no son médicos o médicas forenses ni profesionales de los ministerios públicos. Si bien dichas evaluaciones no son peritajes forenses realizados por expertos en salud mental, sus resultados son ampliamente valorados por los jueces y fiscales al momento de tomar decisiones, como por ejemplo si la persona está o no en condiciones de declarar.

No se conocen investigaciones locales previas que abordan la situación de las personas con discapacidad intelectual y psicosocial en las primeras etapas del proceso penal, particularmente cuando son detenidas por la policía.

El objetivo principal del presente artículo, es determinar qué cantidad de personas con discapacidad intelectual o psicosocial que se encontraban detenidas como sospechosas de la comisión de un delito fueron detectadas por parte del personal médico perteneciente a la policía en la Ciudad de Buenos Aires. Los objetivos secundarios son: a) determinar la cantidad de personas con discapacidad intelectual o psicosocial cuyo diagnóstico era previo a la detención; b) determinar la cantidad de personas con discapacidad intelectual o psicosocial que presentaban certificado de discapacidad previo a su detención; d) determinar qué cantidad de personas con discapacidad intelectual o psicosocial había asistido a una escuela de educación especial; e) determinar si durante las instancias iniciales del proceso se activaron mecanismos de apoyos o ajustes de procedimiento.

6. La obligación de verificar el estado psicofísico de una persona al momento de la detención que se encuentra regulada en el artículo 184 inciso 8 del Código Procesal Penal de la Nación: Los funcionarios de la policía o de las fuerzas de seguridad tendrán las siguientes atribuciones: ... $8^{\circ}$ ) Aprehender a los presuntos culpables en los casos y formas que este Código autoriza y disponer su incomunicación... En tales supuestos deberá practicarse un informe médico a efectos de verificar el estado psicofísico de la persona al momento de su aprehensión.

También se encuentra regulada en el Código Procesal Penal de la Ciudad de Buenos Aires en el artículo 35: Toda persona detenida deberá ser inmediatamente revisada por un médico legista para dejar constancia de su estado físico, la existencia de lesiones y su capacidad para comprender el acto y los hechos que lo motivaron, sin perjuicio de la posterior realización de peritajes al respecto. 


\section{Materiales y Métodos}

Se realizó un estudio observacional de tipo retrospectivo a partir del estudio de registros de 70 casos de personas detenidas en la Ciudad de Buenos Aires por la probable comisión de un delito. Los casos evaluados corresponden a evaluaciones realizadas por el personal médico de la policía entre el 1 de febrero de 2018 y el 18 de octubre de 2019 en las Alcaidías (Comisarías) de la Ciudad de Buenos Aires. De los 70 casos, solo se incluyeron personas con discapacidad intelectual o discapacidad psicosocial - padecimiento psiquiátrico mayor - (trastorno delirante, esquizofrenia, trastorno bipolar). Se excluyeron aquellas personas con trastornos de la personalidad y con trastorno por consumo de sustancias como diagnóstico principal. Todos los casos fueron diagnosticados en forma posterior a la detención por médicos/as psiquiatras forenses con más de cinco años de experiencia específica en el marco de peritajes tendientes a determinar la inimputabilidad y/ o la capacidad para estar en proceso.

\section{Resultados}

Luego de la aplicación de los criterios de inclusión y exclusión la muestra estudiada fue de 47 casos de personas detenidas. De los 70 casos originalmente evaluados, 23 personas fueron excluidas porque no se contaban con toda la información necesaria o no cumplían con los criterios de inclusión en cuanto al diagnóstico.

De los 47 casos analizados (46 eran varones y 1 era mujer y el promedio de edad fue de 30 años) 29 presentaban diagnóstico de discapacidad intelectual y 18 de discapacidad psicosocial (psicosis bajo la forma de esquizofrenia, trastorno delirante). 6 de esas 45 personas fueron evaluadas más de una vez durante el período en estudio con lo cual representaban 61 evaluaciones realizadas por el personal médico de la policía (ver tabla 1).

De las 61 evaluaciones realizadas en solo 5 se realizó la detección de la discapacidad (8\%). Se consideró detección la referencia en el informe médico sobre la repetición de ciclos escolares, antecedentes de internaciones psiquiátricas previas, posesión de un certificado de discapacidad o pensión, o la descripción de síntomas de cuadro psíquico específico (ver tabla 1).

De los 47 casos evaluados 15 (32\%) tenía certificado de discapacidad, 5 (11\%) había asistido a una escuela de educación especial y 26 (55\%) el diagnóstico era previa a su detención (ver tabla 1).

En ninguno (0) de los 47 casos evaluados se han tenido a la vista constancias de la implementación de apoyos y/o ajustes en el procedimiento, tal como lo indica la Convención sobre los Derechos de las Personas con Discapacidad (ver tabla 1). 


\begin{tabular}{|c|c|c|}
\hline & $\begin{array}{l}\text { Cantidad de Personas } \\
\text { evaluadas por el personal } \\
\text { médico de la policía } \\
\text { (N:47) }\end{array}$ & $\begin{array}{l}\text { Cantidad de evaluaciones } \\
\text { realizadas por el personal } \\
\text { médico de la policía } \\
\text { (N:61) }\end{array}$ \\
\hline Género & 46 (masc) 1 (fem) & NA \\
\hline Edad promedio & 30 años & NA \\
\hline Cantidad de personas con discapacidad intelectual & $29(62 \%)$ & NA \\
\hline Cantidad de personas con discapacidad psicosocial & $18(38 \%)$ & NA \\
\hline $\begin{array}{l}\text { Cantidad de personas con certificado de discapacidad } \\
\text { previo a la detención }\end{array}$ & $15(32 \%)$ & NA \\
\hline $\begin{array}{l}\text { Cantidad de personas con diagnóstico previo a la } \\
\text { detención }\end{array}$ & $25(55 \%)$ & NA \\
\hline $\begin{array}{l}\text { Cantidad de personas que asistieron a una escuela de } \\
\text { educación especial }\end{array}$ & $5(11 \%)$ & NA \\
\hline $\begin{array}{l}\text { Cantidad de personas con discapacidad intelectual o } \\
\text { psicosocial detectadas por el personal médico de la } \\
\text { policía }\end{array}$ & NA & $5(8 \%)$ \\
\hline $\begin{array}{l}\text { Cantidad de ajustes de procedimiento y/o de apoyos } \\
\text { realizados }\end{array}$ & 0 & NA \\
\hline
\end{tabular}

Nota: NA - No Aplica.

Fuente: elaboración propia.

\section{Discusión}

Argentina no cuenta con estadísticas actualizadas que permitan conocer la prevalencia de personas con discapacidad intelectual y psicosocial que han entrado en contacto con la justicia penal como acusadas de cometer un delito y/o que se encuentren privadas de libertad en prisión.

Con relación a la prevalencia de personas con discapacidad en la población general un reciente estudio reportó que la prevalencia de población mayor de 6 años con dificultad (Instituto Nacional de Estadísticas y Censos, 2018: 28)7 en Argentina es el 10.2 \% (Instituto Nacional de Estadísticas y Censos, 2018). Específica-

7. En el estudio se definió personas con dificultad a aquellas con al menos una respuesta en las categorías "sí, mucha dificultad" o "no puede hacerlo" en las preguntas sobre dificultades para ver, oír, agarrar y levantar objetos con las manos o los brazos, caminar o subir escaleras, bañarse, vestirse o comer solo/a, comunicarse, aprender cosas, recordar, con- centrarse o controlar su comportamiento y, en particular en el caso de los niños, jugar con niños/as de su edad. Esta definición también incluye a las personas que indicaron usar audífono o tener certificado de discapacidad vigente, hayan respondido o no tener mucha dificultad o no poder hacerlo. Instituto Nacional de Estadísticas y Censos (2018). Estudio Nacional sobre el Perfil de las Personas con Discapacidad: resultados definitivos 2018. Ciudad Autónoma de Buenos Aires: Instituto Nacional de Estadísticas y Censos, p. 28 
mente en lo que se refiere a la discapacidad psicosocial e intelectual ${ }^{8}$, la misma puede estimarse, en forma aproximada, en 0,82 \% de la población entre 15 y 64 años ${ }^{9}$.

Tal como señala la Procuración Penitenciaria de la Nación (Argentina) en su informe anual del año 2018, no hay "información fehaciente, transparente y fidedigna generada por el Estado sobre las personas presas que padecen alguna discapacidad. No existe información pública al respecto." (Procuración Penitenciaria de la Nación, 2018, p. 363) En este sentido el Servicio Penitenciario Federal (SPF) informó que en sus dependencias se encontraban alojadas 80 personas con discapacidad intelectual y 48 con discapacidad psicosocial (Servicio Penitenciario Federal, 2017) ${ }^{10}$. Sin embargo, no se encuentran estadísticas actualizadas al respecto. Empero si trasladamos la prevalencia internacional reportada por la literatura de personas con discapacidad intelectual en prisión (4\%), esto muestra que para el año 2017, 576 personas privadas de libertad en el Servicio Penitenciario Federal y 2558 en todas las cárceles de Argentina (Dirección Nacional de Política Criminal, 2018) tendría una discapacidad intelectual. Cifra muy alejada a la reportada por el Servicio Penitenciario Federal. Tal como se ha señalado Argentina no cuenta con estadísticas actualizadas y confiables sobre las personas con discapacidad intelectual que se encuentra privadas de libertad en la cárcel. En comparación con España, Argentina se encuentra en franca desventaja en esta temática, toda vez que en el caso español ya desde 1994 se han realizado diversos estudios sobre la prevalencia de las personas con discapacidad en el ámbito de la justicia penal (Ramos Feijóo, 2011, p. 9). La Confederación Española de Organizaciones en favor de las Personas con Discapacidad Intelectual (FEAPS, hoy Plena Inclusión) y el Real Patronato de Prevención y de Atención a Personas con Minusvalía (hoy Real Patronato sobre Discapacidad) en 1994 detectaron 419 personas con discapacidad intelectual en 48 establecimientos penitenciarios españoles. Así, la toma de conciencia sobre la situación de este colectivo generó el desarrollo de diversos programas de intervención. En este sentido, estadísticas recientes señalan que en el 2019 el Programa para la Atención de Personas Reclusas y Ex Reclusas con Discapacidad Intelectual llevado adelante por Plena Inclusión, tuvo más de 1700 beneficiarios directos (Araoz Sánchez-Dopico, 2020).

Como puede observarse en el estudio realizado en la Ciudad de Buenos Aires, no existe una identificación adecuada y temprana de las personas con discapacidad en la justicia penal. Esto se presenta como la primera barrera para un adecuado acceso a la justicia. La falta de identificación adecuada y la invisibilización de este colectivo, ya ha sido reportado en previamente (Criminal Justice Joint Inspection, 2015, p. 26).

Más allá de la falta de estadísticas sobre el tema de discapacidad en el contexto penal y penitenciario, nuestro estudio halló que existe una muy deficiente identificación de las personas con discapacidad intelectual y psicosocial por parte del personal médico de policía, solo 5 identificaciones de 61 evaluaciones realizadas. En comparación con el estudio anterior se hallaron resultados similares (Bernardini et al., 2018). Sin embargo, en comparación con lo reportado por Criminal Justice Joint Inspection en 2015, el trabajo aquí realizado mostró resultados mucho más deficientes.

La falta de identificación adecuada de las personas con discapacidad intelectual en el ámbito de la justicia penal y particularmente cuando estas personas son aprehendidas por la policía y se encuentran en Comisaría, ya ha sido previamente reportado por diversos estudios internacionales. En 1997, Bucke y Brown, hallaron

8. En el documento se midió como dificultad cognitiva-mental.

9. Esta cifra es meramente aproximativa toda vez que se toman las cifras poblacionales totales del Censo 2010, en tanto que el estudio realizado sobre personas con discapacidad se realizó a partir de los 6 años.

10. Servicio Penitenciario Federal. Programa De Abordaje Integral Para Personas Privadas De Libertad Con Discapacidad Boletín Público Normativo $\mathrm{N}^{\circ} 641$ 
que solo el $2 \%$ de los detenidos fueron legalmente tratados como personas con discapacidad, cifra que no se corresponde con los reportes internaciones sobre las personas con discapacidad intelectual y psicosocial que se encuentran detenidas (Bucke y Brown, 1997). En una línea similar, Gudjonsson y colaboradores hallaron que solo el $4 \%$ de las personas aprehendidas habían sido identificadas de manera de adecuada por la Policía, en comparación con el $15 \%$ que los autores detectaron. Los autores hallaron que el $15 \%$ de la muestra estudiada presentaba una discapacidad, en tanto que la policía en dicha muestra solo había identificado el $4 \%$. Es decir, la detención por parte de la policía fue inferior a lo reportado por los expertos (Gudjonsson et al., 1993). En 1997 el Informe Mencap halló que solo 38 de 448 personas entrevistadas con discapacidad intelectual fueron adecuadamente reconocidas por la policía (Mencap, 1997). Resultados similares a los obtenidos en el presente estudio. Otro estudio, concluyó que aproximadamente el $50 \%$ de las personas con alguna alteración psíquica fue reportado por la policía (Medford et al., 2003). En resumen, de manera similar a los resultados hallados en el presente trabajo, diversos estudios han reportado la baja detección de personas con discapacidad intelectual o psicosocial en las primeras etapas del proceso (Justice, 1994; Robertson et al., 1996, citado por Medford et al.,2003; Irving y McKenzie, 1989; Medford et al., 2000)

Si bien no es función de los letrados realizar un diagnóstico, sí es posible a través de la entrevista detectar de manera temprana la sospecha de una posible discapacidad intelectual. "(U)na entrevista adecuada puede arrojar datos que permitan deducir, o sospechar...la existencia de una discapacidad intelectual" (Recover y Araoz, 2014, p. 48) En este sentido, los autores señalan que “...sigue siendo fundamental detectar, desde el primer momento, cualquier indicio que permita sospechar de la posible presencia de una (discapacidad intelectual o de desarrollo), y hacerla constar de forma expresa". En este sentido, tanto la Policía (o Cuerpos de Seguridad, en general) como el Letrado, desde las primeras intervenciones que realice en la declaración del denunciado que la presta, deben poner una especial atención a cualquier dato que permita presumir la existencia de una discapacidad o una dificultad cognitiva" (Recover y Araoz, 2014, p. 59). En ese mismo sentido se han desarrollado posibles preguntas para profesionales con el objetivo que pueda ayudarlos a determinar la sospecha de una posible discapacidad intelectual (Plena Inclusión, 2018, p. 108) Por su parte un estudio realizado en Australia por Hayes reportó que los abogados tenían mayor probabilidad de detectar una persona con discapacidad intelectual en comparación con los policías (38,7\% vs 29,5 \% de los casos) (Hayes, 2000, citado por Villamanta, 2012).

Por otra parte, si bien la discapacidad intelectual no es un padecimiento o una enfermedad mental en el ámbito de la justicia suelen ser los psiquiatras y psicólogos forenses quienes, en el contexto de evaluaciones periciales, realizan el diagnóstico en aquellas personas que no tenían un diagnóstico previo a la detención.

Nuestro estudio halló que 15 (32\%) de las personas detenidas por la policía tenía certificado de discapacidad, 5 (11\%) había asistido a una escuela de educación especial y 26 (55\%) el diagnóstico había sido realizado previo a la detención. Estos hallazgos son consistentes con informes previos realizados en España, donde se reportó que más del $50 \%$ de las personas con discapacidad intelectual que se encontraba en la cárcel había recibido previamente el diagnóstico (Defensor del Pueblo, 2019; Araoz Sánchez-Dopico, 2020, p. 52). En tanto que, en Argentina, el Programa Nacional de Asistencia para las Personas con Discapacidad en sus Relaciones con la Administración de Justicia (ADAJUS, 2019) reportó que más del 80 \% de las personas con discapacidad que se encuentran en prisión no tiene certificado de discapacidad. Esta situación plantea el desafío de realizar una detección adecuada a lo largo del todo el proceso penal.

La falta de identificación puede deberse a múltiples causas, como la falta de entrenamiento adecuado en herramientas o preguntas que puedan orientar hacia la presencia de una discapacidad intelectual o psico- 
social, el escaso tiempo de evaluación y de entrevista, las condiciones en las que se realiza la evaluación, las condiciones en las que se encuentra la persona a evaluar. Por ejemplo, el lugar donde se realiza la evaluación (calabozo vs. consultorio médico), con o sin confidencialidad, si el sujeto se encuentra irritable o agresivo, no colabora con el examen, o se encuentra bajo los efectos de alguna sustancia.

Al igual que se ha reportado en trabajos internacionales, el personal médico de la policía en su mayoría son médicos y médicas generales, no psiquiatras y no reciben entrenamiento en este campo, por tal motivo en la mayoría de los casos fallan en detectar a una persona con discapacidad intelectual o psicosocial (Sanders et al., 2010). En Buenos Aires, el personal médico de la policía recibe principalmente formación y entrenamiento en lesionología y en la búsqueda y detección de signos de alteración de la consciencia por una posible intoxicación con sustancias. Situación esta última que llevaría a la solicitud de una derivación a un centro de asistencial.

La falta de entrenamiento en la detección de personas con discapacidad intelectual lleva a que no se realicen preguntas específicas sobre problemas de aprendizaje, repitencias, tipo de escuela a la que concurrió. A su vez ciertas respuestas son muchas veces interpretadas de manera inadecuada. Por ejemplo, no saber o no recordar el número de documento, o fecha de nacimiento es consignado e interpretado como falta de colaboración y no es consignada como que no sabe su número de documento o su fecha de nacimiento o no la recuerda. El no aportar una información requiere primero saber, conocer dicha información y decidir no brindarla, pero no recordar o no saber una información es cualitativamente muy diferente a no quererla aportarla.

En este contexto, es probable que en muchos casos la sospecha de que se está frente a una persona con discapacidad intelectual provenga por parte de los abogados defensores que entrevistan a su asistido unas horas o un día después de la aprehensión, con más tiempo en la entrevista y en muchos casos los abogados defensores han recibido formación básica en la detección de temprana de personas con discapacidad intelectual ${ }^{11}$. Es por ello, que particularmente los abogados defensores que se encuentren sensibilizados en esta temática y realicen preguntas específicas orientadoras, pueden estar en mejores condiciones que el personal médico de policía para sospechar que la persona puede tener una discapacidad intelectual y solicitar una evaluación especializada (Hayes, 2007) En este sentido, el Programa de Reclusos con discapacidad intelectual y del desarrollo realizado por FEAPS (hoy Plena Inclusión) ha señalado la transcendencia de la entrevista de los letrados defensores, que debe ser utilizada como fuente para la recolección de datos que puedan poner en evidencia la presencia de que la persona entrevistada puede tener una discapacidad intelectual (Recover y Araoz, 2014, p. 59)

Las personas con discapacidad intelectual se encuentran fuertemente invisibilizadas en el ámbito de la justicia penal (Bradley, 2009). Se trata de un colectivo altamente vulnerable, tradicionalmente excluido y abandonado socialmente, situación que se ve doblemente agravada dentro del ámbito de la justicia penal (Comisión Interamericana de Derechos Humanos, 2017).

En esta línea, la Comisión Interamericana de Derechos Humanos en forma reciente señaló que “...prisión preventiva afecta de manera desproporcionada a determinadas personas pertenecientes a grupos en situación especial de riesgo" dentro de los que se encuentran las personas con discapacidad (Comisión Interamericana de Derechos Humanos, 2017, p. 21).

11. La Defensoría General de la Nación (Argentina) desarrolló actividades específicas de capacitación y sensibilización sobre las personas con discapacidad intelectual en el ámbito de la justicia penal para defensores públicos. Realizó una breve guía orientadora de preguntas que deben realizar los defensores en las entrevistas con las personas privadas de libertad con el objetivo de obtener datos de interés que puedan orientar a una sospecha fundada de que una persona tiene discapacidad intelectual. 
Particularmente la Comisión señaló que la privación de libertad previa al juicio, afecta de manera desproporcionada a personas que se que encuentran en situación particular de vulnerabilidad, como las personas con discapacidad y especificó que este impacto resulta más grave cuando estas personas se encuentran excluidas económicamente y son víctimas de otras formas de exclusión social. Se requiere en estos casos un enfoque diferenciado, ya que estas personas se encuentran más expuestas a situaciones de riesgo, como ser víctimas de violencia, maltrato, y abuso (Comisión Interamericana de Derechos Humanos, 2017; Rodríguez Rodríguez, 2017, p. 76; United Nations Office on Drugs and Crime, 2009), ya que se trata de un colectivo particularmente vulnerable (Northern Ireland Executive, 2005).

Sin embargo, tal como lo muestran los resultados de este trabajo la falta de identificación adecuada no permite un acceso adecuado a apoyos, ajustes, requerimientos e intervenciones especializadas (Bernardini et al., 2018), como por ejemplo la derivación a un servicio de salud. Asimismo, las intervenciones sociales, educativas y en salud en etapas posteriores del proceso se ven retrasadas o son inexistentes por la falta de detección. Ninguna de las 47 personas con discapacidad intelectual o psicosocial recibió algún tipo de apoyo o ajuste de procedimiento en consonancia con lo que señala el artículo 13 de la Convención sobre los Derechos de la Personas con Discapacidad.

En este sentido, en forma reciente el Comité sobre los Derechos de las Personas con Discapacidad dictaminó que el Estado mexicano violó los artículos 5 (igualdad y no discriminación), 9 (accesibilidad), 12 (igual reconocimiento ante la ley), 13 (acceso a la justicia), 14 (libertad) de la Convención sobre los Derechos de las Personas con Discapacidad, en el caso de una persona con discapacidad psicosocial que fue declarada inimputable, donde se le privó de libertad a través de una medida de seguridad y no se pusieron en marcha apoyos y ajustes que le permitieran acceder a la justicia (Comité sobre los Derechos de las Personas con Discapacidad, 2019).

Debe tenerse presente que la responsabilidad legal y el deber cuidado de una persona que se encuentra privada de libertad recae en el Estado, motivo por el cual resulta de vital importancia detectar en forma temprana si la persona presenta una discapacidad intelectual o psicosocial, y si presenta alguna necesidad especifica o si requiere alguna intervención diferenciada. Así, solo a través de una identificación adecuada se podrán implementar apoyos, ajustes, decidir si se requiere por ejemplo la derivación a un servicio de salud. Asimismo, las intervenciones sociales, educativas y en salud en etapas posteriores del proceso se ven retrasadas o son inexistentes por la falta de detección.

Otra dificultad que se ha detectado es la sobrestimación por parte de los diferentes actores judiciales, principalmente jueces y fiscales de los informes médicos de policía. El personal médico de la policía en la Ciudad de Buenos Aires, y en general en las diferentes jurisdicciones, no cuentan con formación en el modelo de la discapacidad basado en derechos humanos ni con herramientas adecuadas que les permitan la detección e identificación de la discapacidad intelectual y las necesidades de apoyo. Los informes médicos policiales en su gran mayoría solo dan cuenta de la presencia o no de signos de intoxicación por tóxicos, de lesiones corporales y eventualmente de riesgo de daño para sí o para terceros. La ausencia de referencias a una discapacidad intelectual o psicosocial en dichos informes es valorada por los jueces y fiscales como sinónimo de su inexistencia y no como una debilidad de los informes, como consecuencia de una falla en la identificación adecuada. Esto tiene consecuencias en algunos casos graves, ya que al no identificar la discapacidad no se podrán en juego apoyos y ajustes de ningún tipo. Así, se llevarán adelante diferentes instancias procesales, como audiencias, declaraciones, firma de acuerdos, suspensiones de juicio a prueba, o juicios abreviados sin apoyos ni ajustes. Estos hallazgos son coincidentes con investigaciones previas 
realizadas en el ámbito español (Recover y Araoz, 2014, p. 57; Araoz Sánchez-Dopico, 2020, p. 52) Asimismo, se han detectado denegaciones de medidas de prueba fundamentadas en que los informes del personal médico de policía que no hacían referencia a que la persona presentara algún padecimiento mental.

Como se ha señalado, la falta de identificación adecuada trae consecuencias muy negativas para las personas con discapacidad intelectual y psicosocial. La discapacidad permanece invisibilizada y no es posible proponer intervenciones especializadas y específicas que tengan en cuenta la discapacidad que la persona presenta. Se le deniegan así los apoyos, ajustes e intervenciones que pueda requerir ${ }^{12}$. La invisibilización de las personas con discapacidad sometidas a un proceso penal pone de manifiesto que "los operadores jurídicos con los que se ha relacionado la persona durante el proceso (abogados, jueces, Ministerio Fiscal, etc.) no han sido capaces de detectar la existencia de la discapacidad y, por tanto, existe una probabilidad muy alta de que los derechos de estas personas hayan sido vulnerados durante el procedimiento" (Plena Inclusión, 2015, p. 18).

\section{Conclusiones}

Investigaciones internacionales previamente publicadas señalan que las personas con discapacidad intelectual y psicosocial se encuentran sobre representadas en el ámbito de la justicia penal (Søndenaa et al., 2008; Herrington, 2009), motivo por el cual resulta de vital importancia conocer la situación de este colectivo altamente vulnerable en el primer contacto con el sistema penal. Sin embargo, no se conocen investigaciones previas realizadas en Argentina sobre la identificación de personas con discapacidad intelectual y psicosocial por parte del personal policial ni sobre la implementación de ajustes de procedimiento y de apoyos en las primeras etapas del proceso penal.

Tal como lo muestran los resultados del presente trabajo el proceso de detección de personas con discapacidad intelectual, la implementación de ajustes de procedimiento y de apoyos en el ámbito de la justicia penal presenta desafíos pendientes en Argentina. El proceso de detección en las prisiones puede resultar complejo ya sea por los diferentes criterios utilizados, por la falta de recursos, además de la presencia de personas con trastornos mentales y adicciones que pueden enmascarar la presencia de una discapacidad intelectual (Murillo, et al. 2010, p. 56)

El personal policial es el primer punto de contacto entre una persona con discapacidad acusada de una infracción penal y la justicia penal, por tal motivo sus intervenciones cobran gran importancia ya que en la mayoría de los casos marcaran el camino de esas personas (Bradley, 2009). Es por ello que las intervenciones que realice la policía y sus funcionarios en las primeras etapas del proceso son cruciales, dado que brindarán información a los diferentes actores judiciales en las etapas posteriores del proceso (Bradley, 2009).

12. Por ejemplo, una suspensión de juicio a prueba que no tenga en cuenta la discapacidad podría interpretar el incumplimiento de cierta pauta de conducta como por ejemplo la de comparecer mensualmente al juzgado como una causal de revocación de la medida y finalizar con la privación de la libertad. Sin embargo, una persona con discapacidad que no cuenta con una red de apoyo, la falta de cumplimiento puede deberse a que no recuerda dónde queda la oficina, dónde debe presentarse, no sabe qué día, ya que puede tener dificultades para orientarse temporalmente, o no sabe cómo viajar solo a un lugar no familiar para él. Asimismo, una persona con discapacidad intelectual puede tener dificultades para comprender las consecuencias en el largo plazo del incumplimiento de las pautas fijadas, el lenguaje técnico, con palabras como rebeldía puede resultar de difícil comprensión para una persona con discapacidad intelectual. 
Si bien en la Ciudad de Buenos Aires todas las personas que son detenidas reciben una evaluación psicofísica por parte de un médico o una médica de policía, este estudio halló una muy pobre detección de personas con discapacidad intelectual y psicosocial. Esta falta de detección tiene consecuencias tanto para las personas como para su proceso. En las etapas posteriores del proceso, se ha detectado una negación a la realización de estudios periciales psiquiátricos forenses que permitan conocer la influencia de la discapacidad en el hecho, fundando dicha negativa en la ausencia de una discapacidad descripta por el personal médico de la policía. Por otra parte, se ha detectado la firma de juicios abreviados o suspensiones de juicio a prueba, sin ningún tipo de ajuste de procedimiento, además de la exposición a situaciones de maltrato o violencia por parte de otros internos durante la detención.

Asimismo, esta inadecuada detección también se mantiene en otras instancias del proceso penal, como por ejemplo en el ámbito de ejecución de la pena, donde no se acceden a un programa de educación o trabajo adecuada, como así la exposición a situaciones de violencia por parte de otros internos. Esta situación ha sido particularmente destacada por el Comité Español de Representantes de Personas con Discapacidad (CERMI) "...las personas con discapacidad sufren situaciones de maltrato, burla, manipulación, extorsión, intimidación o directamente violencia" (Cuenca Gómez, 2019, p. 156). Asimismo, se ha reportado la mayor reincidencia en conductas antisociales dada la falta de intervención e incorporación a programas que tenga en cuenta la discapacidad (Loucks, 2007).

El presente trabajo se limitó a determinar el porcentaje detección e identificación de personas con discapacidad intelectual y psicosocial acusadas de cometer una infracción penal en la Ciudad de Buenos Aires por parte del personal médico de la policía. Asimismo, se analizaron variables de interés para dicha identificación, tales como el certificado de discapacidad, la escolaridad alcanzada y si se conocía el diagnóstico previo a la aprensión. Por otro lado, se analizó si se implementaron ajustes de procedimiento y/o apoyos durante las primeras etapas del proceso. Al momento de la realización del trabajo no todos los procesos penales habían finalizado, y no fue objeto del estudio analizar el resultado final de cada uno de los casos bajo estudio. Como limitación principal se destaca lo acotado de la muestra analizada (N:61).

La falta de detección adecuada puede deberse a la falta de un protocolo específico para la detección de personas con discapacidad intelectual en el ámbito de las Comisarías y Cárceles, tal como fuera descripto previamente por Talbot (2011)

Se debe mejorar la detección temprana de las personas con discapacidad intelectual en el ámbito penal. En consonancia con recomendaciones internacionales se deben poner en marcha protocolos específicos para garantizar el acceso a la justicia y para ello se requiere mejorar dicha detección (Naciones Unidas, 2020; Bradley, 2009; Ward y Hayes, 2007). Estos protocolos deben realizarse tanto al ingreso de los detenidos en la comisaría como en las cárceles. Cabe resaltar que "...la detección es una condición previa para garantizar los apoyos que la persona precise, esta detección debe ir referida a las necesidades de apoyo y no a la existencia de un diagnóstico o un certificado de discapacidad" (Araoz Sánchez-Dopico, 2020, p. 52). Es muy destacable el trabajo realizado en Argentina por el Programa Nacional de Asistencia para las Personas con Discapacidad en sus Relaciones con la Administración de Justicia (ADAJUS) que en el 2018 ha intervenido en más de 3000 procesos judiciales, $7 \%$ de estos en el ámbito penal (ADAJUS, 2019) ${ }^{13}$. Sin embargo, quedan aún retos pendientes.

13. El programa aborda la situación de todas las personas con discapacidad, y no se encuentra discriminado en qué tipo de discapacidad el Programa ha intervenido específicamente en el ámbito penal. 
Por todo lo expuesto se recomienda en una primera instancia la adopción de una serie de preguntas a realizar por parte del personal médico de la policía que permita orientar hacia la posible presencia de una persona con discapacidad intelectual. Estas preguntas orientadoras no requieren ser realizadas por expertos en salud mental ${ }^{14}$. En una segunda instancia se podrían utilizar pruebas de detección para ser usadas por los oficiales de policía y/o el personal médico de la policía.

Estas medidas de detección serían gran utilidad. No representan un costo elevado ni una sobrecarga excesiva y reportarían grandes beneficios, toda vez que por un lado permitirían derivar a la persona a una evaluación especializada con mayor información y por otro permitirían determinar la necesidad la implementación de apoyos, ajustes o de alguna intervención especial, como por ejemplo un facilitador.

Por otro lado, se recomienda que el personal médico y los funcionarios de la policía reciban capacitación en herramientas para la detección de personas con discapacidad y los derechos que le asisten a este colectivo, particularmente sobre la Convención sobre los Derechos de Personas con Discapacidad (Naciones Unidas, 2020, pp. 27-28). Se ha demostrado que más individuos son reconocidos con discapacidad intelectual cuando el personal policial recibe entrenamiento en este campo (Hayes, 2007). Asimismo, resulta imprescindible realizar capacitaciones continúas y actividades de sensibilización a todos los actores de la justicia penal que permitan cambiar y vencer los obstáculos y barreras actitudinales hacia las personas con discapacidad (Naciones Unidas, 2020, pp. 27-28).

14. Para profundizar sobre buenas prácticas en esta temática se recomienda ver: Recover, T. y Araoz, I. (2014). Las personas con discapacidad intelectual o del desarrollo ante el proceso penal. Análisis sobre la intervención de la persona con discapacidad intelectual o del desarrollo en el proceso penal y el cumplimiento de condenas y medidas de privación de libertad derivadas de este. FEAPS y Plena Inclusión (2018). Acceso a la justicia: ajustes de procedimiento para las personas con discapacidad y del desarrollo. Plena Inclusión. 


\section{Referencias bibliográficas}

Al-Rousan, T. et al. (2017). Inside the nation's largest mental health institution: A prevalence study in a state prison system. BMC public health, 17(1), pp. 1-9.

Araoz Sánchez-Dopico, I. (2020). A cada lado. Informe sobre la situación de personas con discapacidad intelectual reclusas y ex reclusas en España. Plena Inclusión.

Bernardin, L. et al. (2018). Los retos en el acceso de las personas con discapacidad intelectual y psicosocial en el ámbito penal. Estudio comparativo de México y Buenos Aires. Revista Latinoamericana en Discapacidad, Sociedad y Derechos Humanos, 2(2), pp. 51-73.

Bradley, K. (2009). The Bradley Report. Lord Bradley's review of people with mental health problems or learning disabilities in the criminal justice system. https://lx.iriss.org.uk/sites/default/files/resources/The\%20Bradley\%20 report.pdf.

Bucke, T. y Brown, D. (1997). In police custody: police powers and suspects' rights under the revised PACE codes of practice. Home Office Research Series 174. Home Office.

Chaplin, E. et al. (2017). Characteristics of prisoners with intellectual disabilities. Journal of Intellectual Disability Research, 61(12), pp. 1185-1195.

Comisión Interamericana de Derechos Humanos (2017). Informe sobre medidas dirigidas a reducir el uso de la prisión preventiva en las Américas. OEA/Ser.L/VIII.163 Doc. 105. Comisión Interamericana de Derechos Humanos.

Comité sobre los Derechos de las Personas con Discapacidad. (2019). Dictamen aprobado por el Comité en virtud del artículo 5 del Protocolo Facultativo, respecto de la Comunicación núm. 32/2015. CRPD/C/22/D/32/2015, 6 de septiembre de 2019.

Criminal Justice Joint Inspection (2015). A joint inspection of the treatment of offenders with learning disabilities within the criminal justice system - phase two in custody and the community. HMI Probation.

Crocker, A. et al. (2007). Rate and characteristics of men with an intellectual disability in pre-trial detention. Journal of Intellectual \& Developmental Disability, 32(2), pp. 143-152.

Cuenca Gómez, P. (2019). La Adaptación de la Normativa Penitenciaria Española a la Convención Internacional sobre los Derechos de las Personas con Discapacidad. Comité Español de Representantes de Personas con Discapacidad (CERMI). Cinca.

Defensor del Pueblo (2019). Las personas con discapacidad intelectual en prisión. Separata del volumen II del Informe Anual 2018. Defensor del Pueblo.

Dirección Nacional de Política Criminal (2019a). Sistema Nacional de Estadística sobre Ejecución de la Pena. Informe Ejecutivo 2019. https://www.argentina.gob.ar/sites/default/files/informe_sneep_argentina_2019.pdf.

Dirección Nacional de Política Criminal (2019b). Sistema Nacional de Estadística sobre Ejecución de la Pena. Informe del Servicio Penitenciario Federal. https://www.argentina.gob.ar/sites/default/files/informe_sneep_ spf_2019.pdf.

Equipo Adajus (2012). Los derechos de las personas con Discapacidad en situación de encierro, una política de inclusión social intramuros. En O. Rosales (Dir.), Discapacidad, justicia y Estado. Acceso a la justicia de Personas con Discapacidad. Ediciones Infojus. 
Equipo Adajus (2013). Relevamiento de población con discapacidad privada de libertad dentro del Servicio Penitenciario Federal Avances de la primera etapa de investigación. En O. Rosales (Dir.), Discapacidad, justicia y Estado. Discriminación, estereotipos y toma de conciencia (pp. 215-233). Ediciones Infojus.

Fazel, S. et al. (2008). The prevalence of intellectual disabilities among 12000 prisoners. A systematic review. International journal of law and psychiatry, 31(4), pp. 369-373.

Gulati, G. et al. (2018). Intellectual disability in Irish prisoners: systematic review of prevalence. International journal of prisoner health, 14(3), pp. 188-196.

Gudjonsson, G. H. et al. (1993). Persons at Risk During Interviews in Police Custody: The Identification of Vulnerabilities. The Royal Commission on Criminal Justice, Research Study No. 12. HMSO.

Hassiotis, A. et al. (2011). Psychiatric morbidity in prisoners with intellectual disabilities: analysis of prison survey data for England and Wales. The British Journal of Psychiatry, 199(2), pp. 156-157.

Hayes, S. (2007). Missing out: Offenders with learning disabilities and the criminal justice system. British Journal of Learning Disabilities, 35, pp. 146-153.

Herrington, V. (2009). Assessing the prevalence of intellectual disability among young male prisoners. Journal of Intellectual Disability Research, 53(5), pp. 397-410.

Howard, T. y Tyrer, S. (1998). People with learning disabilities in the criminal justice system in England and Wales: a challenge to complacency. Criminal Behavior and Mental Health, 8, pp. 171-177.

Instituto Nacional de Estadísticas y Censos (2018). Estudio Nacional sobre el Perfil de las Personas con Discapacidad: resultados definitivos 2018. Instituto Nacional de Estadísticas y Censos.

Irish Penal Reform Trust (2020). Making rights real for people with disabilities in prison. Irish Penal Reform Trust.

Irving, B. y McKenzie, I. K. (1989). Police interrogation: The effects of the Police and Criminal Evidence Act. Police Foundation of Great Britain.

James, D. y Glaze, L. (2006). Special Report. Mental health problems of prison and jail inmates. NCJ 213600. Bureau of Justice Statistics. https://www.bjs.gov/content/pub/pdf/mhppji.pdf.

Justice (1994). Unreliable evidence? Confessions and the safety of convictions. Justice.

Lizama, V. y Sheinbaum, D. (2020). Hacia un sistema de justicia incluyente: ajustes de procedimiento para personas con discapacidad intelectual y psicosocial. En N. Martínez (Ed.), Salud Mental Forense (pp. 423-443). Tirant lo Blanch.

Lyall, I. et al. (1995). Incidence of persons with a learning disability detained in police custody. A needs assessment for service development. Medicine, Science and the Law, 35(1), pp, 61-71 citado por Howard, T. y Tyrer, S. (1998). People with learning disabilities in the criminal justice system in England and Wales: a challenge to complacency. Criminal Behavior and Mental Health, 8, pp. 171-177.

Lorenzo García, J. (2015). El papel de las familias de las personas con discapacidad intelectual en los procesos de intervención social, ante la comisión de un delito. Una mirada desde el Trabajo Social. https://rua.ua.es/ dspace/bitstream/10045/77529/1/tesis_josefa_lorenzo_garcia.pdf.

Loucks, N. (2007). No one knows: offenders with learning difficulties and learning disabilities. The prevalence and associated needs of offenders with learning difficulties and learning disabilities. Prison Reform Trust. 
Loucks, N. y Talbot, J. (2007). No one knows Identifying and supporting prisoners with learning difficulties and learning disabilities: the views of prison staff. Prison Reform Trust.

Medford, S. et al. (2003). The efficacy of the appropriate adult safeguard during police interviewing. Legal and Criminological Psychology, 8(2), pp. 253-266. https://doi.org/10.1348/135532503322363022.

Mencap Report (1997). Barriers to justice. Mencap.

Mercurio, E. (2016a). Pobreza y discapacidad intelectual en el sistema penal: los invisibles. VERTEX. Revista Argentina de Psiquiatría, XXVII, pp. 197-207.

Mercurio, E. (2016b). Personas con discapacidad intelectual en el sistema penal. Del proceso de normalización a la discriminación. Revista del Ministerio Público de la Defensa de la Nación, 11, pp. 101-110.

Ministerio de Justicia y Ministerio de Salud (2011). Resolución conjunta 1075/2011 y 1128/2011. Créase el Programa Interministerial de Salud Mental Argentino (PRISMA). https://bit.ly/3chMd3H.

Murillo, E. et al. (2010). Detección de personas con discapacidad intelectual en los centros penitenciarios. Revista Española del Tercer Sector, 14, pp. 43-59.

Naciones Unidas (2008). Convención sobre los derechos de las personas con discapacidad. https://www.ohchr. org/SP/HRBodies/CRPD/Pages/disabilitiesconvention.aspx.

Naciones Unidas (2020). Principios y directrices internacionales sobre el acceso a la justicia para las personas con discapacidad. Naciones Unidas.

National Policing Improvement Agency \& Association of Chief Police Officers (2010). Guidance on Responding To People With Mental III Health Or Learning Disabilities. National Policing Improvement Agency.

Northern Ireland Executive (2005). Banford Review of Mental Health and Learning Disability Equal Lives: Review of Policy and Services for People with Learning Disability in Northern Ireland. Northern Ireland Executive. https:// www.health-ni.gov.uk/sites/default/files/publications/dhssps/Equal\%20Lives\%20Report.pdf.

Observatorio estatal de la discapacidad (2018). La situación de la población reclusa con discapacidad en España. https://bit.ly/3fPEAnB.

Organización Mundial de la Salud (2011). Informe Mundial sobre la Discapacidad. OMS.

Pearse, J. (1995). Police Interviewing: The Identification of Vulnerabilities. Journal of Community \& Applied Social Psychology, 5, pp. 147-159.

Plena Inclusión (2015). Informe anual. Plena Inclusión. https://www.plenainclusion.org/sites/default/files/pimemoriadigitalfinal_0.pdf.

Plena Inclusión (2018). Acceso a la justicia: ajustes de procedimiento para las personas con discapacidad y del desarrollo. Plena Inclusión.

Plena Inclusión (2020). La persona facilitadora en procesos judiciales. Plena Inclusión.

Programa Nacional de Asistencia para las Personas con Discapacidad en sus Relaciones con la Administración de Justicia (2019). Informe de buenas prácticas. https://bit.ly/3wSCLfd.

Procuración Penitenciaria de la Nación (2018). Informe Anual: la situación de los derechos humanos en las cárceles federales de la Argentina. Capítulo 3. Personas con discapacidad en prisión. Procuración Penitenciaria de la Nación. 
Ramos Feijóo, C. (2011). Las personas con discapacidad intelectual en el sistema penal- penitenciario. Algunas paradojas de la integración en la exclusión. Las personas con discapacidad intelectual en el sistema penalpenitenciario. Algunas paradojas de la integración en la exclusión. Boletín del Real Patronato sobre Discapacidad, 72, pp. 8-14.

Ramos Feijóo, C. (2013). Las personas con discapacidad intelectual en el sistema penal penitenciario. ¿Un fracaso de las políticas de inclusión? Revista Internacional de Organizaciones, 11, pp. 15-35.

Recover, T. y Araoz, I. (2014). Las personas con discapacidad intelectual o del desarrollo ante el proceso penal. Análisis sobre la intervención de la persona con discapacidad intelectual o del desarrollo en el proceso penal y el cumplimiento de condenas y medidas de privación de libertad derivadas de éste. FEAPS.

Rodríguez, S. (2019). La prisión paso a paso. Versión lectura fácil. Plena Inclusión.

Rodríguez Rodríguez, R. M. (2017). Excluidos e internados. La problemática de las personas con discapacidad en Centros Penitenciarios. Vía Libre. Fundación ONCE. http://www.infocoponline.es/pdf/excluidos_e_internados. pdf.

Sánchez Miranda, M. del C. y Bayona Juan, M. del P. (2019). Discapacidad y prisión. Una reflexión en torno a la privación de libertad en personas con discapacidad intelectual. Antropología Experimental, 19, pp. 81-90.

Sanders, A. et al. (2010). Criminal Justice. Oxford.

Servicio Penitenciario Federal (2017). Programa de abordaje integral para personas privadas de libertad con discapacidad. Boletín Público Normativo, 641.

Søndenaa, E. et al. (2008). The prevalence and nature of intellectual disability in Norwegian prisons. Journal of intellectual disability research, 52(12), pp.1129-1137.

Sheinbaum, D. y Vera, S. (2016). Hacia un sistema de justicia incluyente: proceso penal y discapacidad psicosocial. Documenta. Análisis y Acción para la Justicia Social.

Talbot, J. (2011). Prisoners with learning disabilities and learning difficulties. Prison Service Journal, 195, pp. 29-35.

The National Autistic Society (2008). Autism: a guide for police officers and staff. The National Autistic Society.

The National Autistic Society (2015). Autism: a guide for criminal justice professionals. The National Autistic Society.

Tort, V. et al. (2016). La discapacidad intelectual y el ámbito penitenciario. Revista Española de Sanidad Penitenciaria, 18(1), pp. 25-33.

United Nations Office on Drugs and Crime (2009). Handbook on Prisoners with special needs. United Nations.

Villamanta Disability Rights Legal Service (2012). People who have an Intellectual Disability and the Criminal Justice System. A guide and educational tool for people working in the criminal justice system: Judges, Magistrates, Court Staff, Lawyers, Advocates, Police and Corrections Workers. National Disability Advocacy Program. Australian Government.

Ward, L. y Hayes, S. (Eds.) (2007). Offenders with learning disabilities. British Journal of Learning Disabilities, 35, pp.141-142. https://doi.org/10.1111/j.1468-3156.2007.00463.x.

World Network of Users and Survivors of Psychiatry (2008). Implementation Manual for the United Nations Convention on the Rights of Persons with Disabilities. http://www.wnusp.rafus.dk/documents/WNUSP_CRPD_Manual.pdf. 
Zabala-Baños, M. C. et al. (2016). Mental disorder prevalence and associated risk factors in three prisons of Spain. Revista española de sanidad penitenciaria, 18(1), pp. 13-23. 